\title{
Individualized techniques of Implant Coating with an Antibiotic-loaded, Hydroxyapatite/Calcium sulphate Bone Graft Substitute
}

\author{
Holger Freischmidt ${ }^{1}$, Jonas Armbruster ${ }^{1}$, Gregor Reiter ${ }^{1}$, Paul Alfred Grützner ${ }^{1}$, Lars Helbig ${ }^{2}$ and Thorsten \\ Guehring ${ }^{3 *}$ \\ ${ }^{1}$ BG Unfallklinik Ludwigshafen, Klinik für Unfallchirurgie und Orthopädie, Ludwig-Guttmann-Strasse 13, 67071 Ludwigshafen, Germany \\ ${ }^{2}$ Clinic for Orthopedics and Trauma Surgery, Center for Orthopedics, Trauma Surgery and Spinal Cord Injury, Heidelberg University Hospital, Germany \\ ${ }^{3}$ Arcus Kliniken Pforzheim, Germany
}

Submission: November 19, 2019; Published: December 02, 2019

*Corresponding author: Pilar Aparicio, Upper Limb and Microsurgery Unit, Orthopaedics Department, Parc Sanitari Sant Joan de Déu de Sant Boi de Llobregat, Barcelona, Spain

Abstract

Background: The treatment of fracture- or non-union- related infections has persistently been a major challenge for both patients and treating surgeons. With rising aging of patients and increasing comorbidities, combined with the heterogeneity of germs and any number of multi-resistance against standard antibiotics, a successful treatment is increasingly difficult. One potential solution could be a custom-made individualized antibacterial coating of standard implants with a biphasic degradable biocarrier that releases high doses of antibiotics around the bone-implant-interface. Here we describe our technique of coating intramedullary nails, plates and press-fit shoulder endoprostheses which may prevent bacterial adhesion and biofilm formation. So far, there is very limited experience in individual coating of implants in hip or knee endoprostheses to prevent reoccurrence of surgical-site infection. Currently no reports are available for coating of stems of shoulder prosthesis and nails or plates for fracture fixation.

Methods: Here we show our first experiences with a new individualized surgical technique of coating these implants with a resorbable antibiotic-loaded hydroxyapatite / calcium sulphate biocomposite to prevent biofilm formation and thereby recurrence of bone or joint infection. We describe three cases for coating of plates and nails for fracture fixation and coating of stems of a shoulder prosthesis.

Results: No adverse events of the resorbable bone graft substitute were observed. In all the cases no recurrence of the infection was observed, and osseo-integration was achieved. After implant coating of the shoulder prosthesis no radiological signs of loosening were detected.

Conclusion: We present a new surgical approach of a surface coating of plates, intramedullary nails or prostheses. The osteoconductive- and anti-inflammatory effect of the gentamicin- or vancomycin-loaded hydroxyapatite / calcium sulphate bone graft substitutes shows promising results.

Keywords: Fracture related infections; Osteitis; Osteosynthesis; Local antibiotics; Biodegradable biocomposites

Abbreviations: ALT: Anterolateral free flap; Cerament G: Cerament Antibiotic Loaded with Gentamicin; Cerament V: Cerament Antibiotic Loaded with Vancomycin; CT: Computer tomography; FRI: Fracture Related infection; LCP: Low Compression Plate; LFN: Lateral Femoral Nail; MRSA: Multiresistant Staphylococcus Aureus; PHN: Proxima Humerus Nail; PJI: Periprosthetic joint infection

\section{Background}

Fracture-related infection (FRI) or periprosthetic infection (PJI) remain a major challenge in orthopedic and trauma surgery. Particularly patients with relevant comorbidities such as diabetes, renal failure, peripheral vasculopathy and known nicotine abuse have a high risk for implant- related infections [1]. Numerous devices for local antibiotic application and several treatment options are already available. The aim of these devices is to release local antibiotics in high concentration over a prolonged time period [2], and a new generation of bio-degradable carriers for local antibiotic delivery have been recently emerging for utilization [3]. One of the major difficulties arises from of the ability of microorganisms to adhere to the implant and produce a biofilm layer which unfortunately hinder the treatment of implant-related infections [4-6]. A promising solution could be a commercially available coating of implants such as tibia intramedullary nails. Currently, however the „Expert tibial nail PROtect“(Fa. DePuy Synthes) is the only factory-made antibiotic coated implant available on the market. However, the antibiotics delivery of this coated implant is limited to gentamicin, and it is rather expensive. 


\section{Orthopedics and Rheumatology Open Access Journal (OROAJ)}

As one of the promising biodegradable biocomposites Cerament (supplied by Bone support AB, Lund, Sweden) is an injectable bone graft substitute, composed of $60 \%$ fast resorbing calcium sulphate and $40 \%$ calcium hydroxyapatite [7]. The resorbable local antibiotic carrier provides two modes of actions. It remodels to bone and it provides a local antibiotic concentration which is above the minimal inhibitory concentration (MIC) for most sensitive microorganisms for at least 28 days with - at the same time - safe serum levels [8-10]. The local administration of the antibiotic-containing ceramics promises a better anti-infectious effect than local injection or intravenous infection. Clinical trials demonstrated that Cerament is a viable replacement to bone grafting in filling voids and gaps following fractures [11]. However, in recent literature there is clear evidence of lower infection rates in after additional local antibiotics at least in grade III open fractures [12]. Early histology of Cerament G in Ferguson et al. [3] shows nicely the CERAMENT G covered by an osteoid-like matrix produced by osteoblastic cells seen lining the bone graft substitute's surface. Additionally, Cerament showed good tissue response in an animal model including growth of trabecular bone around the hydroxyapatite particles and complete embedment in bone tissue [13]. Furthermore, Cerament is commercially available with two antibiotic-loaded options. It is marketed as Cerament G (17.5 mg gentamicin sulphate / $\mathrm{ml}$ paste) and Cerament V (66 mg vancomycin / $\mathrm{ml}$ paste) as $5 \mathrm{ml}$ and $10 \mathrm{ml}$ version.

The efficacy of Cerament $G$ and Cerament $V$ in treatment of infected bone defects has already been demonstrated in both osteomyelitis and fracture related infections $[3,14,15]$, as well as in various animal models [16-19]. In an in vitro study the anti-biofilm activity of Cerament $G$ was investigated, suggesting a benefit of this gentamicin-releasing biocomposite for both prophylaxis and treatment of bone infection or implant-related infection, respectively [19]. However, clinical reports on the usage of Cerament G/V as off-label coating device are limited so far. The first clinical experience in using a calcium-based gentamicin or vancomycin loaded bone graft substitute as a surface coating on cement less prosthetic implants has been published by Logoluso et al. In this study $95 \%$ of patients treated for PJI with a twostage revision showed healing without recurrence of infection and no signs of radiographic stem loosening appeared at 12 months follow up [1]. In the here described method we aim to cover each individual implant with Cerament G or Cerament V. This method thus enables to individually coat the most suitable implant (i.e. plates or nails in fracture treatment). In PJI the revision stems of the protheses can be augmented with the most suitable and effective antibiotics (i.e. gentamicin or vancomycin) by augmenting the stem with Cerament G or V. After coating the stem is inserted in a press-fit technique. In the following presentation of our operative technique we provide three new surgical techniques to coat different implants with an antibioticloaded, hydroxyapatite / calcium sulphate bone graft substitutes in order to address the heterogeneity of both microorganism and surgical site.

\section{Methods (Case presentations)}

\section{First Case: Custom-made Implant Coating of a Plate}

A 38-year-old male patient fell of a ride-on mower which then rolled over his left lower leg. This resulted in a second-degree open fracture of the lower limb. Hence, wound debridement and external fixation were performed. The microbiologic results showed an infection with enterobacter intermedius and serratia fonticola. Both microorganisms were sensitive to gentamicin. One month after the accident and several debridements later, the external fixator was removed, and the tibia was stabilized with a low compression plate (LCP). In addition, the tibial defect was filled, and the plate was augmented with $5 \mathrm{ml}$ Cerament $\mathrm{G}$ to prevent infection. Finally, the soft tissue defect was closed with an ALT flap by the plastic surgeons (Figure 1).
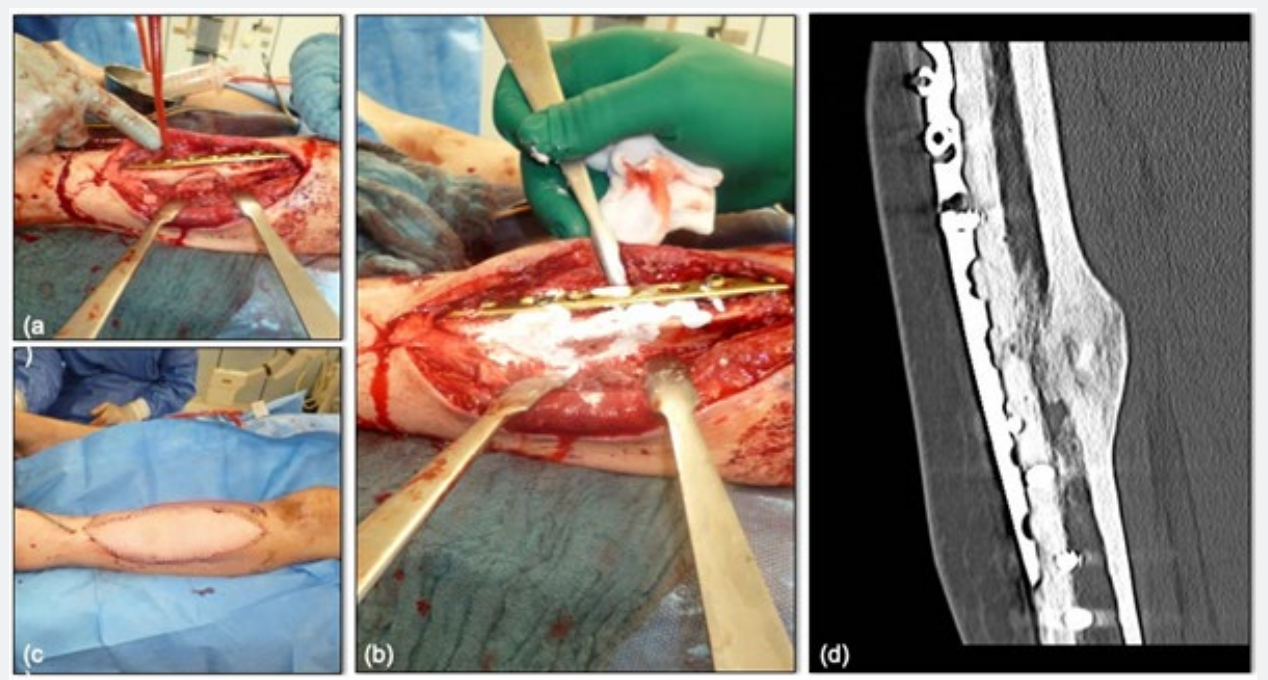

Figure 1: Implant coating of a plate; (a) tibial defect after stabilization with a LCP;

(b) Augmentation of the tibial defect and the plate; (c) after closing the defect with a ALT-flap; (d) bone healing in CT-scan 15 month later. 
Second Case: Implant coating of an intramedullary nail in an infected non-union

A 35-year-old male patient fractured his left femur shaft in a traffic accident in Egypt. Initially, the closed femoral shaft fracture was treated on site with an intramedullary nail. One year later bone healing could not be observed, and the patient still suffered from pain due to a non-union. Therefore, we performed a resection of the non-union and the left femur was immobilized with an external transport fixator as there was a significant shortening of the leg. An infected non-union was diagnosed and a MRSA could be intraoperatively isolated from the non-union site. A bone lengthening was approached for 2 months via the external fixator. However, the lengthening failed due to insufficient stability, and even though a rigid external fixator was subsequently applied for another 6 months, no bone healing could be achieved. Metal removal and complete en bloc resection of the non-union was done and a vancomycin loaded cement spacer was inserted intramedullary. No MRSA was detected during this procedure. As a next step, the cement spacer was removed and a nail-osteosynthesis was inserted after the medullary canal had been reamed (LFN 360/16 mm, Fa Synthes). Before insertion, the nail was augmented with Cerament V (Figure 2b). and the medullary canal was filled up with the remaining amount of Cerament $\mathrm{V}$ using an extension tube A total of 10 millilitres of Cerament $V$ was utilized (Figure 2). Finally, two years after the accident, the nail was dynamically locked for dynamic compression of the non-union. Full weight bearing of the left lower extremity was possible immediately. After 22 months a complete bone healing was achieved (Figure 2c).

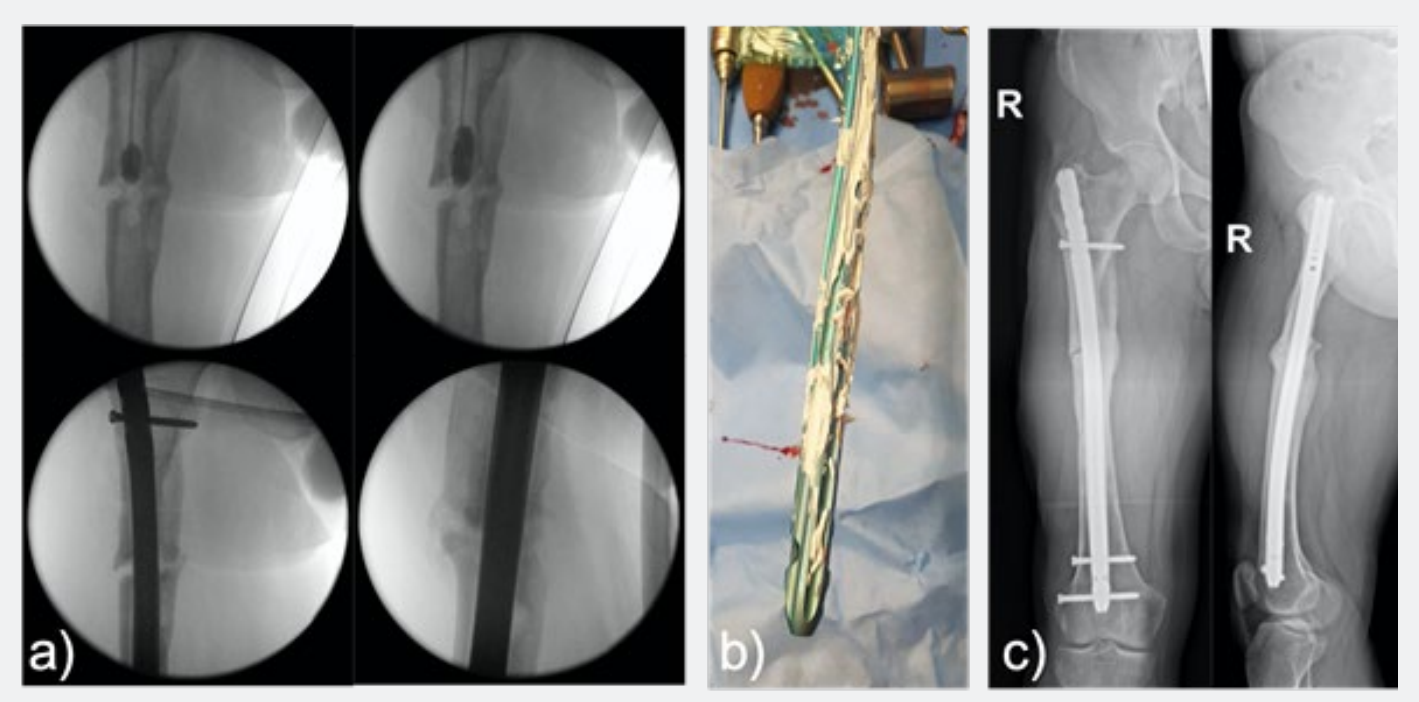

Figure 2: Implant coating of a plate; (a) tibial defect after stabilization with a LCP; (b) Augmentation of the tibial defect and the plate; (c) after closing the defect with a ALT-flap; (d) bone healing in CT-scan 15 month later.

Third Case: Implant coating of a shoulder prosthesis

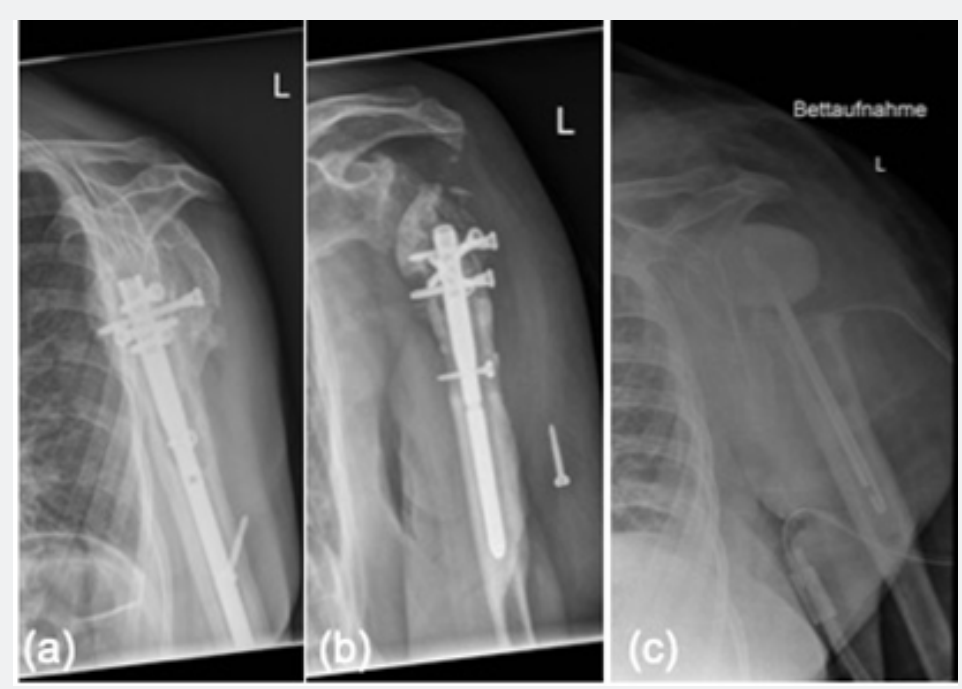

Figure 3: (a) + (b) Removal of the loosened humeral nail; (c) Implantation of a cement spacer. 
A 57-year-old female patient presented with a loosening of a proximal humeral nail (PHN, Fa. Synthes) after fractured left humeral head due to a fall on her shoulder. As reason for the loosening an infection with extensive abscess formation around the implant was identified. The situation was complicated by the patient's incompliance and comorbidities such as schizophrenia. In the first step, we removed the osteosynthesis and implanted a gentamicin and vancomycin loaded cement spacer (Figure 3). Swab examinations showed infection with a staphylococcus lugdunensis, sensitive to vancomycin.
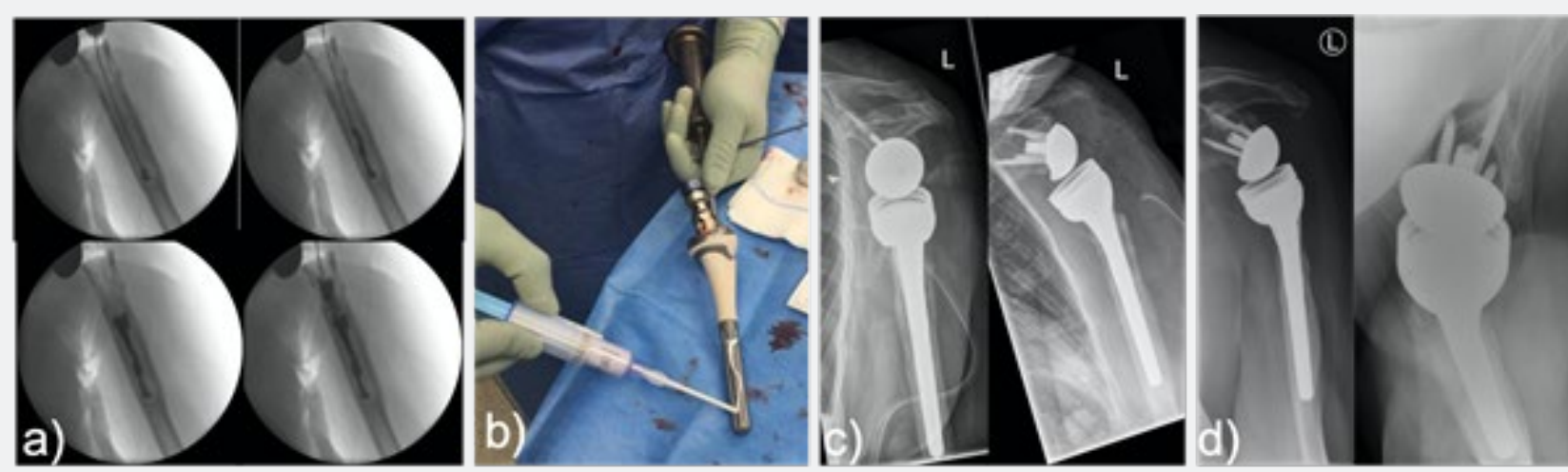

Figure 4: Implant coating of a shoulder prosthesis; (a) Filling the medullary canal with $19 \mathrm{ml}$ Cerament $\mathrm{V}$; (b) coating of the prosthesis with Cerament V; (c) x-ray 2 months after implant coating; (d) x-ray 15 months after implant coating.

During the second revision the shoulder joint was macroscopically free of infection, and thus an implantation of an inverse shoulder prosthesis was done (Fa Synthes DePuy) seven weeks later. During the operation the medullary canal was filled with Cerament V using an extension tube (Figure 4a). In addition, the stem of the prosthesis was augmented (Figure $4 \mathrm{~b}$ ) and thereafter the prosthesis stem was press-fit inserted. A total of 20 milliliters of Cerament $\mathrm{V}$ were used, in accordance with the resistogram. Postoperatively, a dislocation of the prosthesis components occurred. Therefore, at 2 months a proximal enlargement of the stem with an augment was done (Figure 4c). The microbiological samples taken during this surgery were sterile after cultivation (Figure 4).

\section{Results}

So far, there have been no adverse events regarding the use of Cerament $\mathrm{G}$ or $\mathrm{V}$ as a surface coating during the follow up period.

\section{First Case: Implant coating of a plate}

15 months after the augmentation of the LCP with Cerament $\mathrm{G}$ the tibia healed (Figure 1d), the patient is fully weight-bearing without pain. He has a free range of motion in the adjacent joints and no recurrence of the infection has been observed.

\section{Second Case: Implant coating of a nail}

No reinfection occurred 18 months after implantation of the augmented nail-osteosynthesis. 3 months postoperatively the patient could fully weight-bear the right leg with a slightly limping gait remaining. At this time, he did not have pain in the area of non-union and progressing, still incomplete bone healing could be detected in the CT scan. 22 months after surgery a complete bone healing was achieved. The patient is full weight bearing without pain and a free range of motion in the adjacent joints.

\section{Third Case: Implant coating of a shoulder prosthesis}

15 months after implant coating of the shoulder prosthesis with Cerament $\mathrm{V}$ there were no signs for a recurrence of the infection. The shoulder prosthesis was fully integrated. No radiological signs of loosening were detected (Figure $4 \mathrm{~d}$ ). The patient is painless but with limited range of motion (flexion $80^{\circ}$, abduction $70^{\circ}$, external rotation $0^{\circ}$, free range of motion during internal rotation and adduction).

\section{Conclusion}

We present a new surgical approach of an individual off-label surface coating of plates, intramedullary nails or prostheses and could show promising initial results in these cases. To our knowledge this is a relevant innovation in the therapy of fracture- and implant-associated infections. Most importantly no adverse effects of the resorbable bone graft substitute were observed and the osseo-integration of the plates, nails and the prosthesis were observed. However, to gain further knowledge on the osteoconductive- and anti-inflammatory effect of the gentamicin- or vancomycin-loaded hydroxyapatite / calcium sulphate bone graft substitutes, further investigations and prospective clinical studies are required.

\section{Declarations}

Ethics approval and consent to participate. This article does only contain studies with anonymized data; therefore, after inquiry with the ethics commission of the medical association of Rhineland-Palatinate, no ethical approval was needed.

\section{Consent for publication}

We declare that this manuscript is original, has not been published before and is not currently being considered for publication elsewhere. It has been read by all authors and each 
of the authors is convinced that the manuscript represents honest work.

\section{Availability of Data and Materials}

The datasets generated and analysed during the current study are available from the corresponding author on reasonable request.

\section{Competing Interests}

Prof. Dr. med. Thorsten Gühring is a consultant at bone support an at Zimmer Biometd. The remaining authors confirm that there are no conflicts of interest associated with this publication.

\section{Funding}

There has been no financial support for this work that could have influenced its outcome. Non-financial competing interests: no non-financial competing interests exist for any of the authors.

\section{Authors' contributions}

HF and TG participated in the study design and surgeries, analysis of the findings, and drafting of the final manuscript. GR participated in the study design and surgeries. PAG, JA and LH helped draft the final manuscript.

\section{Acknowledgement}

We acknowledge financial support by Deutsche Forschungsgemeinschaft within the funding programme Open Access Publishing, by the Baden-Württemberg Ministry of Science, Research and the Arts and by Ruprecht-KarlsUniversität Heidelberg.

\section{Reference}

1. Logoluso N, Drago L, Gallazzi E, George DA, Morelli I, et al. (2016) Calcium-Based, Antibiotic-Loaded Bone Substitute as an Implant Coating: A Pilot Clinical Study. Journal of bone and joint infection 1: 59-64.

2. Anagnostakos K, Schroder K (2012) Antibiotic-impregnated bone grafts in orthopaedic and trauma surgery: a systematic review of the literature. International journal of biomaterials 2012: 538061.

3. Ferguson J, Athanasou N, Diefenbeck M, McNally M (2019) Radiographic and Histological Analysis of a Synthetic Bone Graft Substitute Eluting Gentamicin in the Treatment of Chronic Osteomyelitis. J Bone Jt Infect $4(2): 76-84$

4. Gristina AG (1987) Biomaterial-centered infection: microbial adhesion versus tissue integration. Science (New York, NY) 237(4822): 15881595.

5. Gristina AG, Naylor P, Myrvik Q (1988) Infections from biomaterials and implants: a race for the surface. Medical progress through technology 14(3-4): 205-224.

6. Gristina AG, Shibata Y, Giridhar G, Kreger A, Myrvik QN (1994) The glycocalyx, biofilm, microbes, and resistant infection. Semin Arthroplasty 5(4): 160-170.
7. Abramo A, Geijer M, Kopylov P, Tagil M (2010) Osteotomy of distal radius fracture malunion using a fast remodeling bone substitute consisting of calcium sulphate and calcium phosphate. J Biomed Mater Res B Appl Biomater 92(1): 281-286.

8. Colding-Rasmussen T, Horstmann P, Petersen MM, Hettwer W (2018) Antibiotic Elution Characteristics and Pharmacokinetics of Gentamicin and Vancomycin from a Mineral Antibiotic Carrier: An in vivo Evaluation of 32 Clinical Cases. J Bone Jt Infect 3(4): 234-240.

9. Stravinskas M, Horstmann P, Ferguson J, Hettwer W, Nilsson M, et al. (2016) Pharmacokinetics of gentamicin eluted from a regenerating bone graft substitute: In vitro and clinical release studies. Bone Joint Res 5(9): 427-435.

10. Stravinskas M, Nilsson M, Vitkauskiene A, Tarasevicius S, Lidgren L (2019) Vancomycin elution from a biphasic ceramic bone substitute. Bone Joint Res 8(2): 49-54.

11. Iundusi R, Gasbarra E, D’Arienzo M, Piccioli A, Tarantino U (2015) Augmentation of tibial plateau fractures with an injectable bone substitute: CERAMENT. Three year follow-up from a prospective study. BMC musculoskeletal disorders 16: 115.

12. Jahangir N, Niazi N, Aljawadi A, Reid A, Wong J, et al. (2019) The use of adjuvant local antibiotic hydroxyapatite bio-composite in the management of open Gustilo Anderson type IIIB fractures. A prospective review. Journal of Orthopaedics 16(3): 278-282.

13. Nilsson M, Wang JS, Wielanek L, Tanner KE, Lidgren L (2004) Biodegradation and biocompatability of a calcium sulphatehydroxyapatite bone substitute. J Bone Joint Surg Br 86(1): 120-125.

14. McNally MA, Ferguson JY, Lau ACK, Diefenbeck M, Scarborough M, et al. (2016) Single-stage treatment of chronic osteomyelitis with a new absorbable, gentamicin-loaded, calcium sulphate/hydroxyapatite biocomposite A prospective Series of 100 Cases. Bone Joint J 98B(9): 1289-1296.

15. Morgenstern M, Vallejo A, McNally MA, Moriarty TF, Ferguson JY, et al. (2018) The effect of local antibiotic prophylaxis when treating open limb fractures: A systematic review and meta-analysis. Bone Joint Res 7(7): 447-456.

16. Dvorzhinskiy A PG, Chojnowski R, Van Der Meulen M, Ross F, Bostrom $M$, et al. (2018) Cerament bone void filler with gentamicin increases bone formation and decreases detectable infection in a rat model of debrided osteomyelitis. Bone Joint J 97(B).

17. Garcia P, Histing T, Holstein JH, Klein M, Laschke MW, Matthys R, et al. (2013) Rodent animal models of delayed bone healing and nonunion formation: a comprehensive review. Eur Cell Mater 26: 1-12; discussion 12-14.

18. Windolf CD, Meng W, Logters TT, MacKenzie CR, Windolf J, et al. (2013) Implant-associated localized osteitis in murine femur fracture by biofilm forming Staphylococcus aureus: a novel experimental model. Journal of orthopaedic research : official publication of the Orthopaedic Research Society 31(12): 2013-2020.

19. Butini ME, Cabric S, Trampuz A, Di Luca M (2018) In vitro antibiofilm activity of a biphasic gentamicin-loaded calcium sulfate/ hydroxyapatite bone graft substitute. Colloids Surf B Biointerfaces 161: $252-260$ 
CC This work is licensed under Creative Commons Attribution 4.0 License

DOI: 10.19080/OROAJ.2019.15.555912

\section{Your next submission with Juniper Publishers} will reach you the below assets

- Quality Editorial service

- Swift Peer Review

- Reprints availability

- E-prints Service

- Manuscript Podcast for convenient understanding

- Global attainment for your research

- Manuscript accessibility in different formats ( Pdf, E-pub, Full Text, Audio)

- Unceasing customer service

Track the below URL for one-step submission https://juniperpublishers.com/online-submission.php 